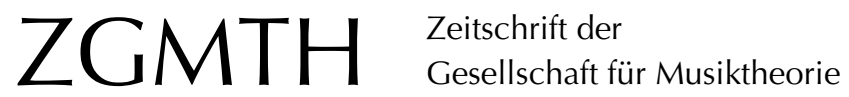

Petersen, Birger (2014): Jan Philipp Sprick, Die Sequenz in der deutschen Musiktheorie um 1900, Hildesheim: Olms 2012 (= Studien zur Geschichte der Musiktheorie 9). ZGMTH 11/1, 149-158. https://doi.org/10.31751/779

(C) 2014 Birger Petersen

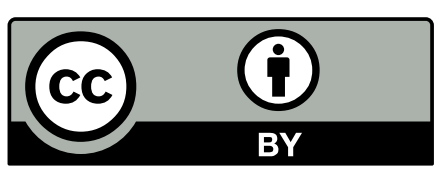

Dieser Text erscheint im Open Access und ist lizenziert unter einer Creative Commons Namensnennung 4.0 International Lizenz.

This is an open access article licensed under a Creative Commons Attribution 4.0 International License.

veröffentlicht / first published: 24/07/2015

zuletzt geändert / last updated: 19/02/2016 


\section{Jan Philipp Sprick, Die Sequenz in der deutschen Musiktheorie um 1900, Hildesheim: Olms 2012 (= Studien zur Geschichte der Musiktheorie 9)}

Was eine Sequenz sei, gehört trotz der Omnipräsenz sowohl des satztechnischen Phänomens in der abendländischen Musikgeschichte der letzten sechshundert Jahre als auch in der musiktheoretischen Literatur seit dem frühen 18. Jahrhundert zu den Fragen, die bislang am wenigsten befriedigend beantwortet wurden: Begriffe wie sSchusterfleckı, Attribute wie smechanisch oder die Beobachtungen Hugo Riemanns zum Verhältnis zwischen Sequenztechnik und Tonalität sind derart selbstverständlich geworden, dass das Grundverständnis von Sequenz nicht mehr hinterfragt wird und eben dadurch eine musiktheoretische Auseinandersetzung mit einem Grundbestand der dur-moll-tonalen Satztechnik verhindert.

Dieser Auseinandersetzung stellt sich Jan Philipp Sprick in seiner 2010 als Dissertationsschrift an der Humboldt-Universität Berlin angenommenen Arbeit. Seine Kernfrage ist weniger eine systematische nach dem Wesen der Sequenz als vielmehr eine historische nach dem, was sie - als satztechnisches Phänomen - den Theoretikern des 19. Jahrhunderts galt; seine Arbeit nimmt sowohl kompositionstechnische Fragestellungen als auch solche der Musikästhetik in den Blick. Nun ist diese Haltung der Disziplin immanent und dem betrachteten Phänomen wesenseigen: Im Fall der Sequenz ist ihre Rezeption in gleichem Maß von der Verknüpfung satztechnischer Aspekte mit ästhetischen Werturteilen bestimmt.

Die Diskussionen, die in den letzten Jahren um historische Satzmodelle geführt wurden, gingen von Dahlhaus' Untersuchungen zur Entstehung der harmonischen Tonalität ${ }^{1}$ aus und sollten die gewonnenen Erkenntnis-

1 Dahlhaus 1968. se vor allem für die praktische Satzlehre und die Analyse nutzbar machen. ${ }^{2}$ Dabei ist auffällig, dass auch die Musik des späteren 19. Jahrhunderts zumeist aus der Perspektive des späten 18. und frühen 19. Jahrhunderts, selten aus einem späteren Blickwinkel, betrachtet wurde. ${ }^{3}$ Sprick hingegen befragt musiktheoretische Lehrwerke zwischen 1870 und 1925 nach ihrem Umgang mit dem Phänomen der Sequenz in der Musik ihrer Gegenwart. Der behandelte Zeitabschnitt ist insofern gut gewählt, als in ihm sowohl diverse parallele Entwicklungen als auch wichtige epochemachende musikgeschichtliche Ereignisse stattfanden. Der Aufbau der Arbeit orientiert sich einerseits an diesen Entwicklungen und Ereignissen, andererseits an der Kontroverse über die ästhetische Bewertung der Sequenz.

Die Leitfragen Spricks betreffen insbesondere die Schnittstelle von Satzlehre und Musikästhetik, für die das Phänomen der Sequenz ein Paradigma darstellt: Akkordprogression, Tonalität und harmonische Funktionalität lassen sich an Sequenzbildungen exemplarisch diskutieren; an ihnen lassen sich neue Theoriebildungen erproben. Entgegen der These Jairo Morenos ${ }^{4}$ zeigt Sprick, dass sich Sequenzen der Einordnung in heutige Analyse-Kategorien (wie Stimmführung und Tonartdarstellung) partiell entziehen können. Es mag unter anderem diese Eigenart der Sequenz gewesen sein, die sie im ausgehenden 19. Jahrhundert kompositorisch interessant gemacht hat und die im frühen 20. Jahrhundert wiederum eine musiktheoretische Reflexion provozierte.

2 Vgl. zu dieser Entwicklung Aerts 2007.

3 Eine Ausnahme bildet Damschroder 2008; vgl. Sprick 2012, 2.

4 Moreno 1996, 83. 
Die Sequenz war Gegenstand satztechnischer und ästhetischer Diskurse. Satztechnische Darstellungen tendieren zu einer negativen Auffassung (die Sequenz wird gewöhnlich als smechanisch abgetan). Demgegenüber haben Vertreter einer (vor allem deutschen) Musikästhetik in ihren Apologien der Sequenz immer wieder die ıSprachähnlichkeitı der Musik und die damit verbundene Möglichkeit der betonenden Wiederholung hervorgehoben. ${ }^{5}$ Schließlich ist in diesem Zusammenhang zu fragen, welchen Beitrag Systemtheorien zum musiktheoretischen Diskurs zu leisten vermögen.

Dem Autor geht es ausdrücklich nicht um eine Theorie der Sequenz - und schon gar nicht unter Berücksichtigung der aktuellen Analyse- und Tonalitätsdiskurse-, sondern um "eine historische Rekonstruktion musiktheoretischen Denkens «. ${ }^{6}$ Das bedeutet, dass Fragen des Sprachgebrauchs und eine Bestandsaufnahme der Terminologie bei der Untersuchung der Schriften eine zentrale Rolle spielen. Es verwundert nicht, dass sowohl die ältere als auch jüngere Forschungsliteratur eine babylonische Vielfalt der Terminologie aufweisen. Um die Untersuchung sinnvoll durchführen zu können, muss auch Sprick systematische Überlegungen anstellen und unterscheidet zwischen einer, Geschichte der Terminologies und deren >Bedeutungsgeschichte ` $^{7} \mathrm{Er}$ verzichtet auf den in der Modelltheorie anderweitig besetzten Begriff des iSequenzmodellss zugunsten des Begriffs 'Sequenzglied und erörtert mit dessen Hilfe die Frage, wie viele Glieder eine Sequenz enthalten muss, um als solche zu gelten. Dabei geht es Sprick nicht darum, diese Frage zu entscheiden, sondern die wechselnden Meinungen der historischen Autoren nachzuvollziehen. ${ }^{8}$

5 Dahlhaus 1984, 433; vgl. Sprick 2012, 4.

6 Sprick 2012, 11.

7 Ebd. 65.

8 Ebd., 5 f.; vgl. Moreno 1996, 6, und Harrison 2003, 226: Harrison unterscheidet zwischen spattern transposition bei einmaliger Wiederholung des Sequenzglieds und spattern sequencer.

\section{Die Sequenz in der ersten Hälfte des}

19. Jahrhunderts

Die einleitenden Kapitel der Arbeit Spricks sind - nach einem Überblickskapitel, das zunächst die negative ästhetische Beurteilung der Sequenz im 19. und frühen 20. Jahrhundert thematisiert - der (vorzugsweise deutschen) Musiktheorie des frühen 19. Jahrhunderts gewidmet (Koch, Schilling, Weber, Marx, Sechter und Fétis). Aufmerksamkeit erfahre das Phänomen spätestens durch Christian Friedrich Daniel Schubart, der in einer Darstellung aus dem Jahr $1774^{9}$ die (zuvor bereits verbürgten) Termini 'Rosalias, sSchusterfleck und ästhetisch bewertet. Anders als Heinrich Christoph Koch, der $1793^{10}$ die Begriffe 'Versetzung`, 'Transposition` und ‘Progression eher satztechnisch und analytisch diskutiert, geht es Schubart in erster Linie um das Aufsuchen von Bewertungskriterien. Dabei spielen in seiner Kritik sogar nationale Ressentiments eine Rolle: So führt er die Namensgebung auf das italienische Volkslied Rosalia, mia cara zurück und schließt sich damit der seinerzeit um sich greifenden Abwertung der italienischen Musik ${ }^{11}$ an (wenngleich Schubart Ausnahmen nennt, etwa Kompositionen von Antonio Caldara). (Vor 'Rosalias und sSchusterfleckı warnt zwar auch Koch, doch billigt er sequenziellen Strukturen eine formale Schlüsselrolle im musikalischen Satz zu.) Die `Vettermicheln schubarts entsprechen den Monte-Bildungen im Sinne Joseph Riepels; dieser wiederum verwendet den Begriff des 'Schusterflecks - anders als die anderen Autoren - nicht für melodische, sondern für harmonische Strukturen. ${ }^{12}$

Die auf Schubart zurückgehende weitgehend negative Bewertung der Sequenz findet sich - ohne dass damals eine theoretische Begründung nachgeliefert wurde - im gesam-

9 Schubart 1774.

10 Koch 1793, insbesondere $206 \mathrm{ff}$.

11 Schubart 1774, 34; vgl. Sprick 2012, 17 f. und $24 \mathrm{f}$.

12 Riepel 1755, 44; vgl. Sprick 2012, 18 ff. 
ten 19. und frühen 20. Jahrhundert, und zwar nicht nur im musiktheoretischen Diskurs: Mit der Darstellung der Kontroverse um die Musik Robert Schumanns im Wagner-Kreis, speziell in einem Text Joseph Rubinsteins, liefert Sprick ein sprechendes Fallbeispiel, das die Breite der Diskussion außerhalb musiktheoretischer Fachdispute abbildet - ein Text, auf den noch 25 Jahre später Guido Adler rekurriert, wenn er 1904 Sequenztechniken in Tristan und Isolde nachweist (37-38). ${ }^{13}$ Arnold Schönbergs Äußerungen wiederum - wie diejenigen Theodor W. Adornos (symptomatisch in dessen Apologetik der Kompositionstechnik Schönbergs) - greifen die ästhetischen Kriterien etwa Schubarts aus dem letzten Drittel des 18. Jahrhunderts wieder auf. In diesem Zusammenhang kommt Sprick am Rande auch auf die Schubert-Rezeption Gustav Mahlers zu sprechen. Sie korrespondiert auf bemerkenswerte Weise mit der Kritik an der Musik Robert Schumanns im Kreis Richard Wagners.

Die Terminologie indes ist einem tiefgreifenden Wandel unterworfen - und fällt zu Beginn des 19. Jahrhunderts zudem zusammen mit der Adjustierung theoretischer Konstruktionen. So findet die Sequenz zwar durchaus eine Würdigung in der Harmonielehre Gottfried Webers, wird bei inm aber trotz aller Neigung zur Systematisierung überkompliziert und verborgen, vor allem aber auch nicht mit einer einheitlichen Chiffrierung belegt: Zwar definiert Weber die Sequenz als »fortgesetzte Reihe einander ähnlicher Harmonieenschritte $\ll^{14}$ und nennt sie als erstes modellhaftes satztechnisches Phänomen noch vor der Kadenz; entsprechend erreicht er auch einen Konnex zwischen der harmonischen Orientierung etwa der Quintfallsequenz und ihrer horizontalen Präsenz in den Einzelstimmen. Dennoch ist die Sequenz - auch in Hinsicht auf die von Weber konsequent gehandhabte Darstellung der Harmonik mit Stufenzeichen - inkonsequent behandelt, was gleichermaßen für die Chiffrierung bei Ernst Friedrich Richter und später bei Hugo

13 Vgl. Adler 1904, 263 f. und 266.

14 Weber 1824, Bd. 2, 177; vgl. Sprick 2012, 49 f.
Riemann gilt. Gleichwohl ist das Urteil Ludwig Holtmeiers einer radikalen »Vertikalisierungs des Verständnisses harmonischer Prozesse" bei Weber ${ }^{15}$, die in der deutschen Musiktheorie des 19. Jahrhunderts weiterwirken sollte, zu differenzieren.

Bei Adolf Bernhard Marx wird der Begriff 'Sequenzı erstmals als eigenständiger musiktheoretischer Terminus verwendet; Marx differenziert im Kontext einer Melodielehre zwischen 'Motiv', 'Gang, und 'Sequenzı. Dabei erscheint die Sequenz - im Sinne einer besonderen Ausprägung der Motivverarbeitung - als spezifische Form des `Ganges ${ }^{16}{ }^{1}$ In seiner Beurteilung, die die Sequenz prinzipiell vor dem Hintergrund des Formdiskurses versteht, wird die variierte Wiederholung ästhetisch höher eingeschätzt als die wörtliche; Theoretiker wie Johann Christian Lobe, Ludwig Bussler und Hugo Riemann knüpfen mehr oder weniger direkt an Marx an.

Demgegenüber wird die Sequenz in Arbeiten, die direkt aus der französischen Tradition des 18. Jahrhunderts erwachsen, oder denen Simon Sechters unter anderen Gesichtspunkten erörtert: Ähnlich wie bei Weber beschreibt auch Charles-Simon Catel die Sequenz als ein kontrapunktisches Phänomen, bei dem sich vertikale und horizontale Aspekte durchdringen. François-Joseph Fétis knüpft an diese Vorstellungen bereits in seinen ersten Annäherungen an den Gegenstand an, wenn er festhält, dass die stonalités durch die Sequenz in Frage gestellt werde. ${ }^{17}$ Dieser wohl meistzitierte Passus aus der Theorie Fétis' wird von Sprick sehr differenziert dargestellt: So sei die Behauptung Fétis', die Sequenz sei ein melodisches und kein harmonisches Phänomen, dahingehend zu modifizieren, dass die Suspendierung der Tonalität nur bei vollkommener Regelmäßigkeit der Stimmführung für den Hörer relevant wird ${ }^{18}$; Fétis zielt dabei vor allem auf die unregelmäßige Behandlung insbesondere der IV. und VII. Stufe.

15 Vgl. Holtmeier 2007a, Sp. 576.

16 Vgl. Marx 1838.

17 Fétis 1844, 27.

18 Sprick 2012, 70-73. 
Fétis erarbeitet seine eigene Kriteriologie einer stonalités als Gegenentwurf zum Fundamentalbassprinzip Jean-Philippe Rameaus; wenn Simon Sechter 1853 in seinen Grundsätzen der musikalischen Komposition ${ }^{19}$ zu einer "orthodoxen Renaissance " der Fundamentalbasstheorie Rameaus gelangt ${ }^{20}$, ist die Breite des Spannungsfelds auch auf der Ebene der für die Fragestellung relevanten Traditionen beschrieben. Auch für Sechter erfüllt die Sequenz vertikale wie horizontale Kriterien: $\mathrm{Er}$ beschreibt mit seiner Kette - durchaus im Sinne Rameaus, wenn auch unter anderen Bedingungen und mit grundsätzlich anderen Schlussfolgerungen - den Zusammenhang zwischen Fundamentschritt und harmonischer Progression, zieht aber den Aspekt der 'Gravitation` dem der dissonanten Septime als Auslöser für die Fortschreitung vor. ${ }^{21}$ Die Theorie Sechters ist äußerst intensiv rezipiert worden, so bei Mayrberger, Bruckner oder Schönberg und sogar Schenker. ${ }^{22}$

\section{'Logikı und >Praxis` der Sequenz}

Das letzte Drittel des 19. Jahrhunderts und die Zeit bis etwa 1925 bilden den zentralen Zeitraum der theoriegeschichtlichen Untersuchung des Autors - zunächst in der Darstellung der Möglichkeiten, die die Musiktheorie Hugo Riemanns mit einer systematischen Integration des Phänomens Sequenz bietet. Sprick verfolgt die Entwicklung der Auseinandersetzung Riemanns mit der Sequenz über vierzig Jahre hinweg und beobachtet dabei den Wandel von primär akustischen Darstellungen über stärker psychologisch geprägte Erklärungsmodelle bis hin zum späten Versuch einer Lehre von den Tonvorstellungen. ${ }^{23}$ Dabei ist die funktionstheoretische Perspektive auf das Phänomen die am stärksten rezipierte, aber bei weitem nicht die einzige, die Riemann gepflegt hat.

19 Sechter 1853.

20 Vgl. Holtmeier 2007b.

21 Sechter 1853, $19 \mathrm{ff} .$, vgl. Sprick 2012, $77 \mathrm{ff}$.

22 Vgl. Sprick 2012, 79.

23 Riemann 1916.
Ausgehend von Riemanns Verständnis einer ımusikalischen Logikı, die Spricks Unterkapitel (»Die ’Logikı der Sequenz ${ }^{24}$ ) den Titel gegeben hat, stellt der Autor Riemanns Sequenzverständnis und die Kritik an dessen Konzept vor, und, die er an zwei Ausschnitten aus den Beethoven-Analysen Riemanns (op. $27 / 1^{25}$ und $53^{26}$ ) konkretisiert. ${ }^{27}$ Sprick legt die zahlreichen Widersprüche in Riemanns Äußerungen zur Sequenz dar - wechselnde ästhetische Bewertungen der Sequenz bei Riemann hängen u.a. von der Textgattung $\mathrm{ab}$ - und kommt zu dem Schluss, dass Riemann »eines der wichtigsten strukturellen Phänomene der tonalen Musik nicht überzeugend in sein systematisches Denken [habe] integrieren [...] können. «" ${ }^{28}$

Als einflussreichen Rezipienten Riemanns führt Sprick August Halm ins Feld, in dessen Harmonielehre ${ }^{29}$ das Thema 'Sequenz im Rahmen eines Exkurses Erwähnung findet; Grundlage für diese Entscheidung ist wiederum Halms eigene, von Riemann abgeleitete Idee einer smusikalischen Logikı: Halm hat deshalb weniger Mühe, das Phänomen der Sequenz in seine Konzeption zu integrieren, weil er die Sequenz der Kadenz nicht nur als Antagonist gegenüberstellt, sondern zu einer analogen sequenziellen ıLogikı kommt. Substantiell abhängig ist diese Konzeption laut Sprick dabei von der Rhetorik-Tradition, ausgehend im 18. Jahrhundert von Mattheson und Koch und im 19. Jahrhundert in Fortsetzung bei Marx. ${ }^{30}$

Mit der Funktionstheorie Hugo Riemanns bildet sich am Ende des 19. Jahrhunderts eine der wirkmächtigsten Theorien heraus; parallel dazu entsteht eine schier unübersehbare Fülle von praxisorientierten Zugängen zur Harmonielehre unterschiedlichsten Zuschnitts. Die

24 Sprick 2014, 83.

25 Riemann (1918-20), Bd. 2, 214 f.

26 Ebd., Bd. 3, 15.

27 Vgl. Sprick 2012, 87-95.

28 Sprick 2012, 118.

29 Halm 1900.

30 Vgl. Sprick 2012, 121. 
in diesem Kontext von Sprick aufgearbeiteten Theoretiker gehören allesamt entweder zum Leipziger Umfeld Riemanns, wie Salomon Jadassohn, Johannes Schreyer oder Hermann van Eyken, oder zu dem der sMünchner Schules Rheinbergers wie Cyrill Kistler oder die Harmonielehre von Rudolf Louis und Ludwig Thuille. Keine nennenswerte Rolle spielt demgegenüber in Spricks Darstellung die Wiener Musiktheorie etwa Arnold Schönbergs oder Heinrich Schenkers; bei dieser Entscheidung beruft sich Sprick auf die Äußerung Dieter Rexroths, die ,Wiener Musiktheorie völlig außerhalb der musiktheoretischen Auseinandersetzungen " gestanden, »die während des 19. Jahrhunderts in Deutschland geführt wurden $\ll^{31}$ - eine Einschätzung, der gerade auf der Basis der jüngeren Publikationen zur Genealogie spezifisch Wiener Traditionen widersprochen werden muss. ${ }^{32}$ Freilich hätte eine Darlegung entsprechender Argumentationen zumal Schönbergs zur Ästhetik der Sequenz (von der oben erwähnten Berücksichtigung im Rahmen des 1. Kapitels abgesehen), vor allem aber eine Auseinandersetzung mit der Theorie Heinrich Schenkers im Kontext dieser Thematik den selbst gesetzten Rahmen gesprengt.

Wenn Sprick die Thematisierung der Sequenz in der zweiten Hälfte des 19. Jahrhunderts, vor allem zwischen 1870 und 1925, unter den Begriffen `Logikı und `Praxis`subsumierend einander gegenüberstellt, geht es ihm in seinem Kapitel zu den praktischen Harmonielehren um andere Leitfragen als in der Auseinandersetzung mit Riemann; das ist überaus nachvollziehbar, hat die Sequenz doch in dieser Gattung musiktheoretischer Publikationen grundsätzlich andere Implikationen als in den systematisch orientierten Darstellungen Rie-

31 Rexroth 1971, 76; vgl. Sprick 2012, $10 \mathrm{f}$.

32 Vgl. z. B. Stefan Rohringer, der die gemeinhin Josef Preindl zugeschriebene und von Ignatz von Seyfried herausgegebene Wiener Tonschule (Wien: Haslinger 1827) als Versuch Seyfrieds begreift, in Rekurs auf die aufkommende Harmonielehre snorddeutsch-protestantischer Prägung »eine Art Gegen-Harmonielehre aus dem Geiste des Generalbasses" zu kreieren $(2012,278)$. manns. Sprick thematisiert hier die Sequenz innerhalb der Erörterung von Akkordprogressionen oder kontrapunktischer Perspektiven des Satzes, hinsichtlich ihrer Chiffrierung und schließlich ihrer Nutzbarmachung als Grundlage klavierpraktischer Übungen. ${ }^{33}$

Die schwache Hierarchisierung stufentheoretischer Ansätze, etwa bei Salomon Jadassohn oder Paul Juon, führt tendenziell zu einer Vernachlässigung tonaler Problemstellungen. Dabei steht Jadassohn sequenziellen Strukturen grundsätzlich sehr skeptisch gegenüber; Juon hingegen räumt - ebenso Kistler, der sich wie Jadassohn auf Richter beruft- der Sequenz einen erheblich breiteren Raum ein. Kistlers Darstellung, die über weite Strecken Generalbass- und stufentheoretische Ansätze miteinander vermengt, wirkt dabei eher unsystematisch, auch in Hinblick auf die Terminologie (»Sequenz, Klimax oder Harmonieverschiebung « $\left.{ }^{34}\right)$; andererseits berücksichtigt Kistler umfänglich die Sequenz als Übungsgegenstand der Klavierpraxis erklärbar aus der Nähe seiner Darstellungen zum Generalbass, aber auch vor dem Hintergrund seiner eigenen Ausbildung bei Rheinberger. ${ }^{35}$ An dieser Stelle ist Spricks Auffassung, die Theorie Kistlers sei in erster Linie der Wagner-Verehrung des Autors geschuldet ${ }^{36}$, zu ergänzen: Der Einfluss des Unterrichts bei Rheinberger ist nicht nur angesichts der im Untertitel der Lehrwerke Kistlers immer wieder evozierten Nähe zum Lehrer offensichtlich. ${ }^{37}$ Die Einflussnahme Wagners einerseits und Rheinbergers andererseits schließen sich allerdings keineswegs gegenseitig aus.

Als entscheidende Differenz zu Riemanns Auseinandersetzung mit dem Phänomen Sequenz in den Arbeiten Kistlers oder Juons kann der Umstand gelten, dass der Zusammenhang zwischen Sequenz und Tonaliät nicht artikuliert wird. Eine Tendenz zur Ver-

33 Vgl. Sprick 2012, $131 \mathrm{ff}$.

34 Kistler 1898, 147; vgl. Sprick 2012, 140 f.

35 Vgl. dazu Petersen 2015.

36 Sprick 2012, 139f. und 144.

37 Vgl. etwa Kistler 1903. 
schmelzung beider Ansätze ist am ehesten für die Arbeit Rudolf Louis' und Ludwig Thuilles zu konstatieren, die in ihrer Harmonielehre ${ }^{38}$ von »funktionalen Paradigmen $«^{39}$ ausgehen, aber an der Stufenchiffrierung Webers festhalten. Von Interesse ist in diesem Zusammenhang aber auch die bislang eher unbeachtete Praktische Harmonielehrevon Paul Juon aus dem Jahr 190140, die in ihrer intensiven Orientierung an der zeitgenössischen kompositorischen Praxis zwar nah an den stufentheoretischen Ansätzen Jadassohns und insbesondere Kistlers (auch und gerade wegen der Betonung der Sequenz als Ausgangspunkt für klavierund kompositionspraktische Übungen), aber eben als systematisch aufgebautes Lehrwerk konzipiert ist.

Auf funktionstheoretischer Seite steht das Lehrbuch der Harmonie und der Elementarkomposition Johannes Schreyers ${ }^{41}$ als Beispiel für die Erörterung der Sequenz im Kontext harmonischer und kontrapunktischer Überlegungen, insbesondere anhand von Literaturbeispielen aus der Klassik und Frühromantik - allerdings ohne der Sequenz einen zentralen Raum zuzuordnen. Wie Schreyers Publikation bewegt sich auch die Harmonielehre Heinrich van Eykens ${ }^{42}$ in einem Grenzbereich zwischen analytisch-systematischem Ansatz und Kompositionspraxis - auch vergleichbar der Wagner-Auseinandersetzung Emile Er$\operatorname{gos}^{43}$, dessen Arbeit allerdings ausschließlich die kompositorische Poetik Wagners betrifft. Was die Integration von Notenbeispielen mit zeitgenössischer Musik betrifft, so finden sich solche bei Autoren, die sich in funktionstheoretischen Grundlagen eher an Riemann orientieren, viel häufiger als bei Riemann selbst. ${ }^{44}$ Die funktionstheoretischen Arbeiten scheinen darin smoderner als die meisten stufentheo-

38 Louis/Thuille 1907.

39 Sprick 2012, 132.

40 Juon 1901.

41 Schreyer, Johannes 1924.

42 Eyken 1911.

43 Ergo 1914.

44 Vgl. etwa Louis/Thuille 1907. retischen: So verzichtet etwa Jadassohn vollständig auf Literaturbeispiele.

Die Preisgabe des harmonischen Dualismus zugunsten einer monistischen Variante der Funktionstheorie, die für praktische Harmonielehren konstitutiv ist, bildet die Basis für Ludwig Holtmeiers These, dass Riemann »keine Schule ausbilden« hätte können. ${ }^{45}$ Diese Annahme wird von Sprick dahingehend differenziert, dass einerseits eine Vielzahl von Autoren mehr oder weniger Bezug auf Riemann nehme; außerdem existiere mit der Neo-Riemannian Theory mittlerweile sehr wohl eine Art 'Schule ${ }^{46}$ : Die Bedeutung der Tonnetze insbesondere für das späte Denken Riemanns sei in der deutschsprachigen Riemann-Rezeption konsequent ausgeblendet worden, während sie in der jüngeren amerikanischen Musiktheorie zur Kenntnis genommen werde. ${ }^{47}$

\section{Ausblick: Von Capellen zu Güldenstein}

In der Rezeption Riemanns durch Hermann Grabner und Wilhelm Maler erfährt die Sequenz eine nahezu vollständige Eliminierung aus dem musiktheoretischen Diskurs, wie Sprick bereits in seinem zentralen Kapitel über Hugo Riemann abschließend darstellt ${ }^{48}$; Grabner ersetzt gar in seinem Handbuch der Harmonielehre den historisch verbürgten und überaus üblichen Terminus der Quintfallsequenz durch den der »QuartschrittSequenz«. ${ }^{49}$

Neuartige Ansätze in der Musiktheorie bzw. im Bereich der Analyse - auch und speziell in der Harmonielehre - führen nach 1900 allerdings auch zu einem Wandel der ästhetischen Bewertung von Sequenzen. Zum Teil werden die mit dem 19. Jahrhundert überkommenen Urteile auf den Kopf gestellt, weil das präsentierte Material zum Bezugspunkt einer auf die zeitgenössische Kompositionspraxis gerichte-

45 Holtmeier 2005, 230; vgl. Sprick 2012, 146 f.

46 Sprick 2012, 146 und 148-151.

47 Vgl. dazu auch Sprick 2014.

48 Sprick 2012, 126-129.

49 Grabner 1944, 93 f.; vgl. Sprick 2012, 126 f. 
ten Musiktheorie wird; als Beispiel heranzuziehen ist die Rezeption der Werke Richard Wagners in der zeitgenössischen Musikkritik.

Die nunmehr tendenziell positive ästhetische Bewertung der Sequenz beschreibt Sprick - in Anlehnung an August Halm - als sdritte Kultur der Sequenz: Im Mittelpunkt eines umfang- und inhaltsreichen letzten Abschnitts steht die Wahrnehmung des Phänomens bei Georg Capellen, Ernst Kurth, Hermann Erpf und Gustav Güldenstein, also der Generation der Musiktheoretiker nach Riemann. ${ }^{50}$ Ihren Anfang findet die grundsätzlich andere Beurteilung mit Halms eigener, bereits erwähnter Rede von der 'Logik der Sequenz;; die Autoren dieser Gruppe eint dabei die prinzipielle Frage nach dem ästhetischen Potential der Sequenz jenseits einer meist pejorativen Wertung - im Kontext einer Wendung zur theoretischen Harmonielehre, auch dokumentiert in den spekulativen Ansätzen etwa Capellens oder Güldensteins: Georg Capellen erarbeitet die Sequenz unter dem Aspekt der sSymmetrie und weist ihr in seinem theoretischen Denken eine zentrale Rolle zu, während Gustav Güldenstein sie mit dem Begriff der Gravitation assoziiert. Anders noch als diese und ihre Vorgänger entwickeln Ernst Kurth und Hermann Erpf unter Bezugnahme auf die Musik Anton Bruckners ein neues terminologisches Repertoire.

Der Aspekt der Symmetrie, der daneben auch in den musiktheoretischen Beiträgen Bernhard Ziehns ${ }^{51}$ und Hermann Schroeders ${ }^{52}$ eine Rolle spielt, ist Ausgangspunkt für die Darstellung der Sequenz bei Georg Capellen: Ausgehend von einer eigenen Spielart des Dualismus, den Capellen trotz seiner erklärten Gegnerschaft zu Hugo Riemann aufgreift, erarbeitet er sogar ein eigenes harmonisches Bezeichnungssystem, das sich allerdings ebenso wenig wie das ursprüngliche System Riemanns durchsetzen konnte; Sprick erklärt zunächst konzise die Grundlagen der Theorie Capellens, ohne die ein Verständnis von des-

50 Sprick, 2012, 167-208.

51 Ziehn 1888.

52 Schroeder 1902. sen Perspektive auf die Sequenz nicht oder nur sehr eingeschränkt möglich sein dürfte. ${ }^{53}$ In seiner Fortschrittliche[n] Harmonie- und Melodielehre von 1908 kehrt Capellen die These Fétis', die Sequenz suspendiere die Tonalität bzw. stelle sie grundsätzlich in Frage, anhand einer leitereigenen Sequenz aus einer Elegie Edvard Griegs um: ${ }^{54}$ Auf der Basis seiner Theorie der »Modellnachahmung" - im Zusammenwirken von Harmonik und Melodik - stellt er für eine Sequenz dasjenige fest, was er anderorts "Einheit der Tonart « nennt. ${ }^{55}$ Capellen fordert sogar die Ablösung leitereigener Harmoniesysteme - Symmetrie realisiert sich für ihn in der realen Sequenzierung. Der Gedanke Capellens - so Sprick - sei hier, dass der tonale Zusammenhang eine reale Sequenz gewissermaßen in eine leitereigene Form »zwinge «.$^{56}$

Mit August Halm, Ernst Kurth und schließlich Hermann Erpf gerät die Debatte um den Wert von Sequenzbildungen in neues Fahrwasser: Halm verweist in seiner BrucknerMonographie und der Apologie des von ihm hochverehrten Komponisten die Abwertung der Sequenz mit den Begriffen 'Schusterflecks und 'Rosalies in die »Rumpelkammer der Aesthetik ${ }^{57}$ - für Sprick ein deutliches Indiz für einen grundsätzlichen Bedeutungswandel im Umgang mit dem satztechnischen Phänomen. ${ }^{58}$ So verteidigt Ernst Kurth die Sequenz mit einer Neubewertung des der Sequenz innewohnenden sMechanischen - ein gebräuchlicher Vorwurf, der demnach nur aus der kompositionstechnischen, nicht aber aus einer musikästhetischen Perspektive eine Berechtigung haben kann. Dabei ist auffällig, dass er den Begriff ,Sequenz` als »melodisches Bruchstück « versteht ${ }^{59}$ - weniger die harmonische als die melodische Ebene leitend

53 Sprick 2012, 172-175.

54 Vgl. Capellen 1908, 87.

55 Capellen 1904, 52; vgl. Sprick 2012, 176 ff.

56 Sprick 2012, 183.

57 Halm 1914, 186.

58 Sprick 2012, 187.

59 Kurth 1923, 334. 
ist. Eine besondere Rolle kommt der Sequenz bei der "Durchbrechung" der Tonalität, die er anhand von Wagners Tristan und Isolde untersucht, zu: Kurth betrachtet wie Capellen die reale Sequenz als Normalfall. Dabei bildet Kurth keine systematischen Kategorien mehr, wie er denn auch in seinen Analysen von Sequenzen auf Stufenbezifferungen verzichtet und Sequenzglieder nur mit eher nivellierenden Klammern bezeichnet. Im Rahmen seiner Bruckner-Analysen werden sequenzielle Strukturen mit den Begriffen "Wellenzüge» und "Wellensteigerungen " belegt ${ }^{60}$, was die Aufgabe dieser Strukturen im formalen Kontext dokumentiert. Sprick kommt an dieser Stelle zu einer erhellenden Darstellung der Formästhetik bei Kurth, die durchaus auf satztechnischen Ereignissen fuße und zudem (und damit verbunden) der Sequenz eine zentrale Rolle zubillige. ${ }^{61}$

Hermann Erpf schließlich - auch als Kritiker der Theorie Kurths - ist bemüht, die Funktionstheorie seines Lehrers Riemann mit dem Denken Kurths und Halms zu verbinden; in seinen Studien zur Harmonie- und Klangtechnik der neueren Musik von 1927 thematisiert er die Sequenz erstaunlicherweise erstmals in einem Kapitel mit dem Titel »Funktionslose Zusammenklänge « ${ }^{62}$, weil er davon ausgeht, dass selbst bei einer tonalen Sequenz eine Abfolge funktionsfreier Harmonieverbindungen vorliegt. (Erpf nimmt symmetrische Klangbildungen zum Ausgangspunkt des Kapitels - bemerkenswert angesichts der Rolle, die Capellen und Zeitgenossen der Symmetriebildung im Kontext der Sequenz zugesprochen haben.) Seine Differenzierung zwischen »leitertreue Verschiebung" und »intervalltreue Sequenz« nimmt vor allem Bezug auf romantische Sequenzbildungen ${ }^{63}$; sein Hinweis auf die damit verbundene dynamische Formgliederung untermauert die Zugehörigkeit Erpfs zu einer Gruppe mit Kurth - allerdings in einer Mittlerposition.

60 Ebd., 335.

61 Sprick 2012, $192 \mathrm{f}$.

62 Erpf 1927, 72-86; vgl. Sprick 2012, 195.
Die Äußerungen Gustav Güldensteins zur Sequenz in dessen Theorie der Tonart von 1928 erscheinen bei Sprick als Grenzpunkt einer spekulativen Musiktheorie: Güldensteins Erörterung der ins Unendliche fortgesetzten Quintfallsequenz, der »kosmisch[en] « Sequen $z^{64}$, scheint philosophischen Ursprungs, ist aber in enger Bezugnahme unter anderem auf Riemann, Stumpf, Capellen, Kurth und Schenker erklärbar; ihre Anwendungen sowohl in Beispielen als auch in konstruierten Modellen verweisen stärker als die Arbeiten von Güldensteins Zeitgenossen auf das Musikschaffen etwa Regers oder Richard Strauss'. Der Wert Güldensteins liegt Sprick zufolge so vor allem im Aufgreifen der entscheidenden Diskussionen ${ }^{65}$, aber - so mag ergänzt werden -auch im Fortschreiben der Auseinandersetzung mit der kompositorischen Praxis.

Die brillante Studie Jan Philipp Spricks weist deutlich über das konkrete Problem der Sequenz in der Musiktheorie des späten 19. Jahrhunderts hinaus: So bietet die Publikation eine Reihe von möglichen Anknüpfungspunkten für weitere Untersuchungen - sowohl im Bereich der Geschichte der Musiktheorie, etwa in der Aufarbeitung des Phänomens 'Sequenz in der Perspektive des 20. Jahrhunderts nach Grabner als auch hinsichtlich der analytischen Betrachtung von Musik aus dem Blickwinkel der zeitgenössischen Musiktheorie und ganz allgemein in der Einbettung von musikästhetischen Fragestellungen in satztechnische Erörterungen und umgekehrt. Das Phänomen 'Sequenz ist bei weitem kein unscheinbares Problem, sondern erweist sich in der Zusammenschau von kompositionstechnischen wie musikästhetischen Perspektiven als paradigmatischer Gegenstand - für die Geschichte der Musiktheorie ebenso wie für grundsätzliche musiktheoretische Fragestellungen.

Birger Petersen

63 Erpf 1927, $76 f$.

64 Güldenstein 1928, 15.

65 Sprick 2012, $207 f$. 


\section{Literatur}

Adler, Guido (1904), Richard Wagner. Vorlesungen gehalten an der Universität zu Wien, Leipzig.

Aerts, Hans (2007), "sModell und `Topos` in der deutschsprachigen Literatur seit Hugo Riemann«, ZGMTH 4/1-2, Hildesheim u. a.: Olms, 143-158.

Capellen, Georg (1904), Die Freiheit der Töne und Intervalle als Kriterien der Stimmführung, nebst einem Anhang: Grieg-Analysen als Bestätigungsnachweis und Wegweiser der neuen Musiktheorie, Leipzig: Kahnt.

- (1908), Fortschrittliche Harmonie- und Melodielehre, Leipzig: Khant.

Dahlhaus, Carl (1968), Untersuchungen über die Entstehung der harmonischen Tonalität, Kassel u.a.: Bärenreiter, Neuedition in: ders., Gesammelte Schriften, hg. von Hermann Danuser, Bd. 3, Laaber: Laaber 2001, 11-307.

- (1984), Die Musiktheorie im 18. und 19. Jahrhundert. Erster Teil: Grundzüge einer Systematik (= Geschichte der Musiktheorie 10), Darmstadt: Wissenschaftliche Buchgesellschaft, Neuedition in: ders., Gesammelte Schriften, hg. von Hermann Danuser, Bd. 4, Laaber: Laaber 2002, 237-410.

Damschroder, David (2008), Thinking about Harmony. Historical Perspectives on Analysis, Cambridge: Cambridge University Press.

Erpf, Hermann (1927), Studien zur Harmonie- und Klangtechnik der neueren Musik, Leipzig: Breitkopf \& Härtel.

Eyken, Heinrich van (1911), Harmonielehre, hg. und bearbeitet von Hugo Leichtentritt und Oskar Wappenschmitt, Leipzig: Hofmeister.

Ergo, Emile (1914), Ueber Wagner's Harmonik und Melodik, Leipzig: Breitkopf \& Härtel.

Erpf, Hermann (1927), Studien zur Harmonie- und Klangtechnik der neueren Musik, Leipzig: Breitkopf \& Härtel.

Fétis, François-Joseph (1844), Traité complet de la théorie et de la pratique de I'harmonie, Paris: Schlesinger.
Güldenstein, Gustav (1928), Theorie der Tonart, Stuttgart: Klett.

Grabner, Hermann (1944), Handbuch der Harmonielehre, Berlin: M. Hesse.

Halm, August (1900), Harmonielehre, Leipzig: Göschen.

- (1914), Die Symphonie Anton Bruckners, München: Müller.

Harrison, Daniel (2003), »Rosalia, Aloysius and Arcangelo: a Genealogy of the Sequence", Journal of Music Theory 47/2, 225-272.

Holtmeier, Ludwig (2005), „Grundzüge der Riemann-Rezeption", in: Musiktheorie (= Handbuch der Systematischen Musikwissenschaft Bd. 2), hg. von Helga de la Motte-Haber und Oliver Schwab-Felisch, Laaber: Laaber, 230-262.

- (2007a), Art. "Gottfried Weber", in: Die Musik in Geschichte und Gegenwart, 2. Aufl., hg. von Ludwig Finscher, Personenteil Bd. 17, Kassel/Stuttgart: Bärenreiter und Metzler, 574-577.

- (2007b), »Heinichen, Rameau, and the Italian Thoroughbass Tradition: Concepts of Tonality and Chord in the Rule of the Octave «, Journal of Music Theory 51/1, 5-49.

Juon, Paul (1901), Praktische Harmonielehre [Lehrbuch und Aufgabenbuch], Berlin: Schlesinger.

Kistler, Cyrill (1898), Harmonielehre, 2., sehr vermehrte Auflage, Heilbronn: Schmidt.

- (1903), Der einfache Contrapunkt und die einfache Fuge. (System Rheinberger-Kistler), Heilbronn: Schmidt.

Koch, Heinrich Christoph (1793), Versuch einer Anleitung zur Composition, Bd. 3, Leipzig: Adam Friedrich Böhme, Reprint Hildesheim u. a.: Olms 2000.

Kurth, Ernst (1923), Romantische Harmonik und ihre Krise in Wagners Tristan, 3. Auflage, Berlin: Hesse.

Louis, Rudolf / Thuille Ludwig (1907), Harmonielehre, Stuttgart: Grüninger.

Marx, Adolf Bernhard (1838), Art. "Sequenz", in: Encyklopaedie der gesamten musikalischen Wissenschaften oder Universal-Lexi- 
kon der Tonkunst, Bd. 6, hg. von Gustav Schilling, Stuttgart: Köhler, 338-339.

Moreno, Jairo (1996), Theoretical Reception of the Sequence and its Conceptual Implications, Ph. D. Dissertation, Yale University.

Petersen, Birger (2015), »Rheinbergers Bassübungen für Harmonielehre und die Partimento-Tradition im 19. Jahrhundert«, in: Musiktheorie im 19. Jahrhundert. Bericht über den XI. Kongress der Gesellschaft für Musiktheorie Bern 2011 (Musikforschung der Hochschule für Künste Bern), hg. von Martin Skamletz, Michael Lehner und Stephan Zirwes, Bern: Argus [im Druck].

Rexroth, Dieter (1971), Arnold Schönberg als Theoretiker der tonalen Harmonik, Phil. Diss. 1969, Bonn.

Riemann, Hugo (1916), »Ideen zu einer 'Lehre von den Tonvorstellungen «, in: Jahrbuch der Musikbibliothek Peters 21/22, Leipzig: Peters, 1-26.

- (1918-20), L. van Beethovens sämtliche Klaviersonaten. Ästhetische und formaltechnische Analyse mit historischen Notizen, 3 Bde., Berlin: Hesse.

Riepel, Joseph. 1755. Grundregeln zur Tonordnung insgemein. Abermal durchgehends mit musicalischen Exempeln abgefaßt und Gesprächs-weise vorgetragen, Frankfurt a.M., Leipzig, Regensburg: Bader.

Rohringer, Stefan (2012), „Franz Schubert, die Wiener Generalbasslehre seiner Zeit und die historisch informierte Analyse «, in: Im Schatten des Kunstwerks - Komponisten als Theoretiker in Wien vom 17. Jahrhundert bis Anfang 19. Jahrhundert (= Wiener Veröffentlichungen zur Theorie und Interpretation der Musik Bd. 1), Wien: Praesens, 273-297.

Schreyer, Johannes (1924), Lehrbuch der Harmonie und der Elemtarkomposition, 5. vollständig umgearbeitete und vermehrte Auflage, Leipzig: Merseburger.

Schröder, Hermann (1902), Die symmetrische Umkehrung in der Musik (= Publikationen der Internationalen Musikgesellschaft 8), Leipzig: Breitkopf \& Härtel.

Schubart, Christian Friedrich Daniel (1774), "Von den Rosalien«, in: Deutsche Chronik 1/3, Dritte Beylage, 33-38.

Sechter, Simon (1853), Die Grundsätze der musikalischen Komposition, Bd. 1, Leipzig: Breitkopf \& Härtel.

Sprick, Jan Philipp (2012), Die Sequenz in der deutschen Musiktheorie um 1900 (= Studien zur Geschichte der Musiktheorie 9), Hildesheim u. a.: Olms.

- (2014), »Reger, Riemann und die neo-riemannian theory", in: Musiktheorie und Vermittlung (= Paraphrasen - Weimarer Beiträge zur Musiktheorie 2), hg. von Ralf Kubicek, Hildesheim: Olms, 277-285.

Weber, Gottfried (1824), Versuch einer geordneten Theorie der Tonsetzkunst, 2. Aufl., 4 Bde., Mainz: Schott.

Ziehn, Bernhard (1888), Harmonie- und Modulationslehre, Berlin: Vieweg. 